\title{
Breast cancer outcome prediction with tumour tissue images and machine learning
}

\author{
Riku Turkki ${ }^{1,11}$ (1) - Dmitrii Byckhov ${ }^{1} \cdot$ Mikael Lundin $^{1} \cdot$ Jorma Isola $^{2} \cdot$ Stig Nordling $^{3} \cdot$ Panu E. Kovanen ${ }^{4}$. \\ Clare Verrill $^{5,6} \cdot$ Karl von Smitten $^{7} \cdot$ Heikki Joensuu $^{8} \cdot$ Johan Lundin $^{1,9} \cdot$ Nina Linder $^{1,10}$
}

Received: 10 December 2018 / Accepted: 16 May 2019 / Published online: 22 May 2019

(c) The Author(s) 2019

\begin{abstract}
Purpose Recent advances in machine learning have enabled better understanding of large and complex visual data. Here, we aim to investigate patient outcome prediction with a machine learning method using only an image of tumour sample as an input.

Methods Utilising tissue microarray (TMA) samples obtained from the primary tumour of patients $(N=1299)$ within a nationwide breast cancer series with long-term-follow-up, we train and validate a machine learning method for patient outcome prediction. The prediction is performed by classifying samples into low or high digital risk score (DRS) groups. The outcome classifier is trained using sample images of 868 patients and evaluated and compared with human expert classification in a test set of 431 patients.

Results In univariate survival analysis, the DRS classification resulted in a hazard ratio of 2.10 (95\% CI 1.33-3.32, $p=0.001$ ) for breast cancer-specific survival. The DRS classification remained as an independent predictor of breast cancer-specific survival in a multivariate Cox model with a hazard ratio of 2.04 (95\% CI 1.20-3.44, $p=0.007$ ). The accuracy (C-index) of the DRS grouping was 0.60 (95\% CI 0.55-0.65), as compared to 0.58 (95\% CI 0.53-0.63) for human expert predictions based on the same TMA samples.

Conclusions Our findings demonstrate the feasibility of learning prognostic signals in tumour tissue images without domain knowledge. Although further validation is needed, our study suggests that machine learning algorithms can extract prognostically relevant information from tumour histology complementing the currently used prognostic factors in breast cancer.
\end{abstract}

Keywords Breast cancer $\cdot$ Machine learning $\cdot$ Deep learning $\cdot$ Outcome prediction

Electronic supplementary material The online version of this article (https://doi.org/10.1007/s10549-019-05281-1) contains supplementary material, which is available to authorized users.

Riku Turkki

riku.turkki@helsinki.fi

1 Institute for Molecular Medicine Finland (FIMM), University of Helsinki, Helsinki, Finland

2 Department of Cancer Biology, BioMediTech, University of Tampere, Tampere, Finland

3 Department of Pathology, Medicum, University of Helsinki, Helsinki, Finland

4 HUSLAB and Medicum, Helsinki University Hospital Cancer Center and University of Helsinki, Helsinki, Finland

5 Nuffield Department of Surgical Sciences, University of Oxford, Oxford, UK

Abbreviations
AUC Area under receiver operating characteristics

CI $\quad$ Confe
CNN $\quad$ Convolutional neural network

6 NIHR Oxford Biomedical Research Centre, Oxford, UK

7 Eira Hospital, Helsinki, Finland

8 Department of Oncology, Helsinki University Hospital and University of Helsinki, Helsinki, Finland

9 Department of Public Health Sciences, Karolinska Institutet, Stockholm, Sweden

10 Department of Women's and Children's Health, International Maternal and Child health (IMCH), Uppsala University, Uppsala, Sweden

11 Science for Life Laboratory (SciLifeLab), Karolinska Institutet, Solna, Sweden 


$\begin{array}{ll}\text { DSS } & \text { Disease-specific survival } \\ \text { DRS } & \text { Digital risk score } \\ \text { ECW } & \text { Enhanced wavelet compression } \\ \text { ER } & \text { Oestrogen receptor } \\ \text { GMM } & \text { Gaussian mixture model } \\ \text { HER2 } & \text { Human epidermal growth factor receptor 2 } \\ \text { HR } & \text { Hazard ratio } \\ \text { IFV } & \text { Improved Fisher vector } \\ \text { OS } & \text { Overall survival } \\ \text { PCA } & \text { Principal component analysis } \\ \text { PR } & \text { Progesterone receptor } \\ \text { SVM } & \text { Support vector machine } \\ \text { TIL } & \text { Tumour-infiltrating lymphocytes } \\ \text { TMA } & \text { Tissue microarray }\end{array}$

\section{Background}

There is a growing interest around the potential of machine learning to improve the accuracy of medical diagnostics [1]. Novel machine learning techniques have not only advanced the state-of-the-art in several pattern recognition tasks [2,3], but also have the potential to extract clinically relevant information from complex medical imaging data sets. Especially, methods using deep learning have been successful in various medical image analysis tasks $[4,5]$, some of them reaching performance of experienced specialists in individual diagnostic tasks $[6,7]$.

Within pathology, whole-slide scanners have enabled accurate digitisation of histological samples with sub-micrometre resolution allowing for computerised analysis of the specimens with machine learning algorithms [8]. Computerised methods for detection of mitoses [9-11], infiltrating immune cells $[12,13]$ and other tissue entities such as segmentation of epithelial and stromal tissue compartments or discrimination between viable and non-viable tissue [14-18] have been studied. Recent reviews [5, 8, 19] offer thorough summaries on methods developed for analysis of histological samples.

Specific type of deep learning methods, convolutional neural networks (CNNs), is frequently used in the development of the image-based classifiers. CNNs are composed of consecutive, interconnected and hierarchical stages-an architecture inspired by the structure of biological neural networks [20]—-making it a powerful tool to capture abstract patterns in visual data. Utility of CNNs was recently demonstrated in detection of breast cancer metastases in lymph node tissue sections [21].

Instead of predicting patient outcome based on intermediate quantification of tissue structures, such as specific cell types and states (e.g. mitotic cells, pleomorphic cells, immune cells) or tissue structures and entities (ducts, necrosis, vessels), our aim in this study is to predict patient outcome based solely on the visual appearance of the breast cancer tissue without any prior assumptions. We hypothesise that a method capable of inferring relevant signals for outcome without prior knowledge of tissue structures may be able to reveal complementary and unbiased prognostic information.

\section{Materials and methods}

\section{Patients and preparation of tumour tissue microarrays}

We pooled two data sets for the study, the FinProg series and a similar single-centre series from Helsinki University Central Hospital. The FinProg series $(N=1860)$ is a nationwide cohort including approximately $50 \%$ of all women diagnosed with breast cancer in Finland 1991 and 1992 [22] and cover most of the patients (93\%) within five selected geographical regions (the FinProg Breast Cancer Database ${ }^{1}$ ). The other patient series $(N=527)$ consists of patients diagnosed mainly in the Helsinki region and treated at the Department of Surgery and Oncology, Helsinki University Hospital, from 1987 to 1990. Both series include comprehensive information on clinical and pathologic characteristics extracted from the hospital and laboratory records. In the current study, we used information on histological grade and type, tumour size, number of positive lymph nodes, patient age, as well as oestrogen (ER), progesterone (PR) and human epidermal growth factor receptor 2 (HER2) status. In addition, we had information on treatment type given; $62 \%$ of the patient received local therapy and $42 \%$ systemic therapy.

Routinely fixed paraffin-embedded breast cancer samples were retrieved from the archives of pathology laboratories, and representative tumour regions identified for preparation of TMA blocks [23]. From the tissue blocks available, three representative $0.60 \mathrm{~mm}$ tissue cores were punched and assembled into 23 TMA blocks, each containing 50-144 tumour tissue cores. Immunohistochemistry, chromogen in situ hybridisation, as well as grading [24] were performed as previously described [22].

Inclusion criteria for the current study were the following: survival data with cause of death, images of the breast tumour tissue, as well as a tissue sample area $>0.02 \mathrm{~mm}^{2}$ (corresponding to 400,000 pixels in the image). Patients with lobular or ductal carcinoma in situ, synchronous or metachronous bilateral breast cancer or other malignancy (except for basal cell carcinoma or cervical carcinoma in situ), distant metastasis, or who did not undergo surgery of the primary tumour were excluded. In addition, the TMAs

\footnotetext{
${ }^{1}$ http://www.finprog.org.
} 
that were checked for quality and non-representative, samples without tumour tissue, were excluded. After exclusions, 1299 tissue spots, one per patient, were available for further analysis. Lastly, the spots were randomly divided into separate training $(N=868,67 \%)$ and test $(N=431,33 \%)$ sets. Compared to the commonly used 80-20\% split, we assigned more samples (33\%) to the test set in order to also enable subgroup and multivariate analyses. The median follow-up of patients in the final patient cohort alive at the end of follow-up period is 15.9 years (range 15.0-20.9, interquartile range $15.4-16.3$ years).

\section{Image acquisition}

Five-micrometre thick sections were cut from the TMA blocks, stained with haematoxylin and eosin and digitised with a whole-slide scanner (Pannoramic 250 FLASH, 3DHISTECH Ltd., Budapest, Hungary) equipped with a $20 \times$ objective (numerical aperture 0.80 ) and a $1 \times$ adapter, and a progressive scan colour camera with three separate charge-coupled devices with $1618 \times 1236$ pixels sized $4.40 \mu \mathrm{m} \times 4.40 \mu \mathrm{m}$ (CIS_VCC_F52U25CL, CIS Corporation, Tokyo, Japan) resulting in an image where one pixel represents an area of $0.22 \mu \mathrm{m} \times 0.22 \mu \mathrm{m}$. Images were stored in a whole-slide image format (MRX, 3DHISTECH Ltd., Budapest, Hungary) and further compressed to a wavelet file format (Enhanced Compressed Wavelet, ECW, ER Mapper, Intergraph, Atlanta, GA) with a compression ratio of 1:10. The compressed virtual slides were uploaded to a wholeslide image management server (WebMicroscope, Aiforia Technologies Oy, Helsinki, Finland) where individual images of TMA spots were segmented from the whole-slide image and downloaded for algorithm training and testing as uncompressed portable network graphics files.

\section{Outcome classification}

We extracted local convolutional image descriptors for each TMA spot image by reading the activations from the last convolutional layer of convolutional neural network (VGG-16) trained on the ImageNet database [25], and used improved Fisher vector (IFV) encoding [26] to aggregate the descriptors from the image foreground regions into a single descriptor. The network used (VGG-16) is a 16-layer network with small $3 \times 3$ convolutional filters. The network was not trained or fine-tuned on our data set, instead we used it only as a feature extractor. A benefit of the descriptor aggregation approach is that an image of arbitrary size can be given as an input for the model. In addition, a study [27] showed that descriptor aggregation with IFV might yield stronger discrimination when compared to fully connected layers. For computation of the IFV, the convolutional image descriptors were compressed with principal component analysis (PCA) from 512 channels into 16 components, and 64 mixture components were used in quantising the data with a Gaussian mixture model (GMM). We defined the image foreground regions by applying Otsu's thresholding [28] to a transformed spot image $I_{t}$, when $I_{t}=\left(\ln \left(1-I_{g}\right)+2 e\right) / 2 e$, and where $I_{g}$ is a Gaussian-filtered (radius of 15 pixels) grayscale version (averaged over colour channels) of the spot image. After the thresholding, all objects smaller than 12,500 pixels in area were removed. Finally, we compressed the IFV descriptors with PCA into 48 bins before classification with a linear support vector machine (SVM). The analysis pipeline was implemented in a numerical computing environment (MATLAB R2016b, MathWorks, Natick, MA, U.S.) using libraries for computer vision and machine learning [29-31].

For training the DRS group classifier, we defined two categories according to the patients' survival status and follow-up time. In the first category (high risk, $N=340$ ), we included all the patients who died due to breast cancer earlier than 10 years after the diagnosis, and in the other category (low risk, $N=528$ ), we included the patients who did not die of breast cancer during the entire follow-up time. For learning the unsupervised IFV encoding, we randomly sub-sampled $4 \times 10^{6}$ local image descriptors from the training set. The sampling was balanced between low-risk and high-risk spots. In training the SVM model, we used 868 breast cancer TMA spot images, each spot representing an individual patient.

\section{Visual risk scoring}

Three pathologists scored the test set TMA spot images into low and high-risk groups using a web-based viewing and annotation software (WebMicroscope, Aiforia Technologies Oy, Helsinki, Finland). Prior and during the visual scoring, the pathologists were able to view the training set TMA spots grouped as they were labelled in training of the SVM classifier. Based on the pathologists' scoring, a visual risk score (high risk or low risk) was formed with majority voting. Furthermore, one pathologist assessed the following tissue entities in each TMA spot: mitoses ( 0 vs. 1 vs. $>1)$, pleomorphism (minimal vs. moderate vs. marked), tubules ( $\leq 10$ vs. $10-75$ vs. $>75 \%$ ), necrosis (absent vs. present) and quantity of tumour-infiltrating lymphocytes (TILs) (low vs. high).

\section{Statistical analysis}

The Kaplan-Meier method was used for estimating the survival function [32] and the log-rank test was used in comparison of survival curves. The disease-specific survival (DSS) time was defined as the time period between date of diagnosis and death of breast cancer, censoring patients who were 


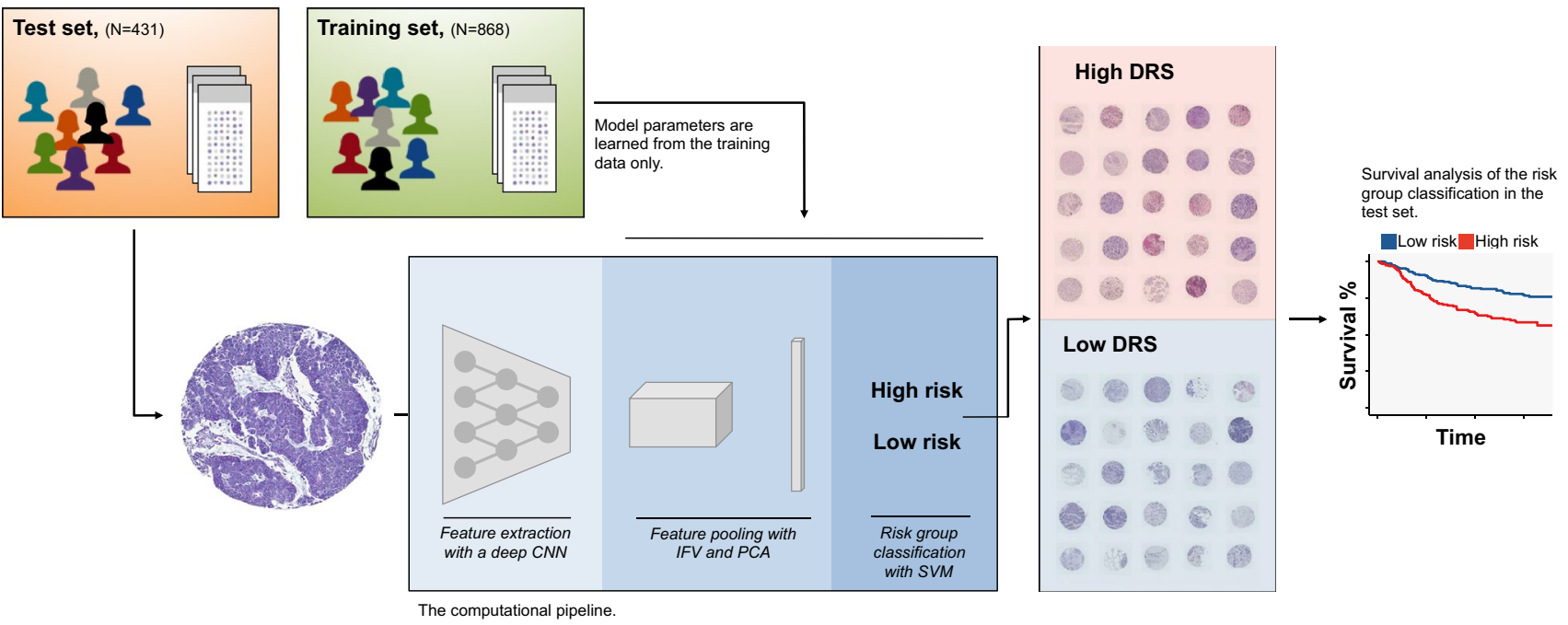

Fig. 1 Workflow for training and testing the digital risk score (DRS) classification. The computational pipeline consists of three sequential steps: (i) feature extraction with a deep convolutional neural network (CNN), (ii) feature pooling with improved Fisher vector encoding

alive on the date of the last contact, and those who had died from another cause on the date of death. The overall survival (OS) time was defined as the time period between the date of breast cancer diagnosis and death of any cause, censoring patients alive on the date of the last contact. For estimating the effect size (hazard ratio, HR) while accounting for the effect of other covariates, we used the Cox proportional hazard model [33]. C-index (concordance) and AUC were used to evaluate the discrimination and prediction accuracy of survival models [34]. Chi-squared contingency table test was used for comparison of categorical variables, and continuous distributions were compared with Kruskal-Wallis test. All statistical analyses with a two-sided $p$ value lower than 0.05 were considered significant.

\section{Results}

\section{Outcome classification}

We trained the outcome classifier using a training set of 868 tumour tissue images, and subsequently classified the test set representing 431 breast cancer patients into low and high DRS groups (Fig. 1). In the test set, 237 (55\%) patients were classified into the low DRS group and 194 (45\%) patients into the high DRS group. The patient characteristics are summarised in Table 1. The DRS model performance rates measured with area under receiver operating characteristics curve (AUC) on the test and training sets were 0.58 and 0.63 , respectively, indicating no substantial model overfitting (Supplementary Fig. S1).
(IFV) and principal component analysis (PCA) and (iii) classification with support vector machine (SVM). Training set samples are used in supervision and a separate test set-up used in validation

\section{Outcome classification and clinicopathological variables}

Patients predicted to have an increased risk of breast cancerspecific death had significantly greater proportion of highgrade tumours $(p=0.014)$ as compared to patients who were assigned to the low DRS group (Table 1). Moreover, patients in the high DRS group had larger tumours $(p<0.001)$, higher number of positive lymph nodes $(p=0.003)$ and were more often PR-negative $(p=0.015)$.

\section{Outcome classification and survival analysis}

We investigated the prognostic value of the DRS grouping with univariable and multivariable survival analysis in the test set. Women in the lower DRS group had more favourable breast cancer-specific $(p<0.001)$ and overall survival $(p=0.003$ ) (Fig. 2). Ten-year DSS in the low DRS group was $82 \%$ (95\% CI 77-87\%) compared to $65 \%$ (95\% CI 58-73\%) in the high DRS group. When the cancers were split according to histological grade assessed from original whole-slide samples, the DRS grouping showed the strongest discrimination in grade I cancer $(\mathrm{P}<0.001)$, whereas the differences observed in grade II $(p=0.410)$ and grade III $(p=0.083)$ groups were not statistically significant (Fig. 3). When the cancers were divided according the steroid hormone receptor status, the DRS classifier was a significant predictor of survival both in the ER positive $(p=0.025)$ and ER negative $(p<0.001)$ subgroups. The DRS grouping was a significant predictor in the PR-negative subgroups, but not in the subset of PR-positive breast cancer $(p=0.003)$. Furthermore, 
Table 1 Patient characteristics

\begin{tabular}{|c|c|c|c|c|c|c|c|c|c|c|}
\hline \multirow[t]{3}{*}{ Variables } & \multicolumn{5}{|c|}{ Whole data set $(N=1299)$} & \multicolumn{5}{|c|}{ Test set $(N=431)$} \\
\hline & \multicolumn{2}{|c|}{$\begin{array}{l}\text { Training set } \\
(N=868)\end{array}$} & \multicolumn{2}{|c|}{$\begin{array}{l}\text { Training set } \\
(N=431)\end{array}$} & \multirow[t]{2}{*}{$p$ Value } & \multicolumn{2}{|c|}{$\begin{array}{l}\text { Low DRS } \\
(N=237)\end{array}$} & \multicolumn{2}{|c|}{$\begin{array}{l}\text { High DRS } \\
(N=194)\end{array}$} & \multirow[t]{2}{*}{$p$ Value } \\
\hline & $\%$ & $N$ & $\%$ & $N$ & & $\%$ & $N$ & $\%$ & $N$ & \\
\hline \multicolumn{11}{|c|}{ Number of positive lymph nodes } \\
\hline Mean & 1.4 & & 1.2 & & 0.407 & 0.9 & & 1.6 & & 0.003 \\
\hline 0 & 58 & 504 & 59 & 253 & 0.323 & 63 & 150 & 53 & 103 & 0.057 \\
\hline $1-3$ & 24 & 206 & 23 & 99 & & 23 & 54 & 23 & 45 & \\
\hline $4-9$ & 8 & 73 & 9 & 38 & & 6 & 15 & 12 & 23 & \\
\hline$>10$ & 3 & 30 & 2 & 7 & & 1 & 2 & 3 & 5 & \\
\hline Unknown & 6 & 55 & 8 & 34 & & 7 & 16 & 9 & 18 & \\
\hline \multicolumn{11}{|c|}{ Tumour size (per mm) } \\
\hline Mean & 23.7 & & 23.2 & & 0.817 & 2.15 & & 25.3 & & $<0.001$ \\
\hline Unknown & 3 & 28 & 5 & 22 & & 5 & 13 & 5 & 9 & \\
\hline \multicolumn{11}{|l|}{ Histological grade } \\
\hline I & 16 & 143 & 19 & 83 & 0.086 & 23 & 54 & 22 & 43 & 0.014 \\
\hline II & 34 & 296 & 36 & 154 & & 32 & 75 & 41 & 79 & \\
\hline III & 23 & 197 & 18 & 76 & & 14 & 33 & 22 & 43 & \\
\hline Unknown & 27 & 232 & 27 & 118 & & 32 & 75 & 22 & 43 & \\
\hline \multicolumn{11}{|l|}{ Histological type } \\
\hline Ductal & 76 & 662 & 77 & 333 & 0.742 & 74 & 175 & 81 & 158 & 0.079 \\
\hline Lobular/special & 24 & 206 & 23 & 98 & & 26 & 62 & 19 & 36 & \\
\hline \multicolumn{11}{|l|}{ Age } \\
\hline$\leq 39$ & 7 & 63 & 7 & 30 & 0.353 & 9 & 21 & 5 & 9 & 0.140 \\
\hline $40-49$ & 21 & 186 & 24 & 103 & & 27 & 64 & 20 & 39 & \\
\hline $50-59$ & 27 & 234 & 22 & 94 & & 21 & 49 & 23 & 45 & \\
\hline $60-69$ & 20 & 172 & 21 & 91 & & 20 & 47 & 23 & 44 & \\
\hline$\geq 70$ & 25 & 213 & 26 & 113 & & 24 & 56 & 29 & 57 & \\
\hline \multicolumn{11}{|l|}{ ER } \\
\hline Negative & 29 & 248 & 27 & 116 & 0.572 & 25 & 60 & 29 & 56 & 0.443 \\
\hline Positive & 62 & 538 & 64 & 274 & & 65 & 155 & 61 & 119 & \\
\hline Unknown & 9 & 82 & 10 & 41 & & 9 & 22 & 10 & 19 & \\
\hline \multicolumn{11}{|l|}{ PR } \\
\hline Negative & 42 & 362 & 41 & 177 & 0.803 & 36 & 86 & 47 & 91 & 0.015 \\
\hline Positive & 49 & 423 & 50 & 215 & & 56 & 132 & 43 & 83 & \\
\hline Unknown & 10 & 83 & 9 & 39 & & 8 & 19 & 10 & 20 & \\
\hline \multicolumn{11}{|l|}{ HER2 } \\
\hline Negative & 72 & 623 & 74 & 321 & 0.713 & 76 & 181 & 72 & 140 & 0.136 \\
\hline Positive & 17 & 146 & 16 & 70 & & 14 & 32 & 20 & 38 & \\
\hline Unknown & 11 & 99 & 9 & 40 & & 10 & 24 & 8 & 16 & \\
\hline
\end{tabular}

Left association of clinicopathological variables in the training and test sets. Right: association of clinicopathological variables between patients in low and high digital risk score (DRS) groups

$p$-values $<0.05$ are shown in bold

the risk grouping was a significant predictor for survival both among HER 2 negative $(p=0.015)$ and positive patients $(p<0.001)$. Subgroup analysis according to tumour size and nodal status are shown in Figure 4.

A multivariate survival analysis showed that classification into the high DRS group was associated with unfavourable prognosis $(\mathrm{HR}=2.04,95 \%$ CI $1.20-3.44, p=0.007)$, and indicated that the DRS grouping is an independent predictor for survival (Table 2). Tumour size (1.04, 95\% CI 1.02-1.06, $p<0.001)$, PR positivity $(0.42,95 \%$ CI $0.25-0.71, p<0.001)$ and having $>10$ positive lymph nodes $(\mathrm{HR}=4.74,95 \% \mathrm{CI}$ $1.17-19.30, p<0.029)$ were also independent predictors. 

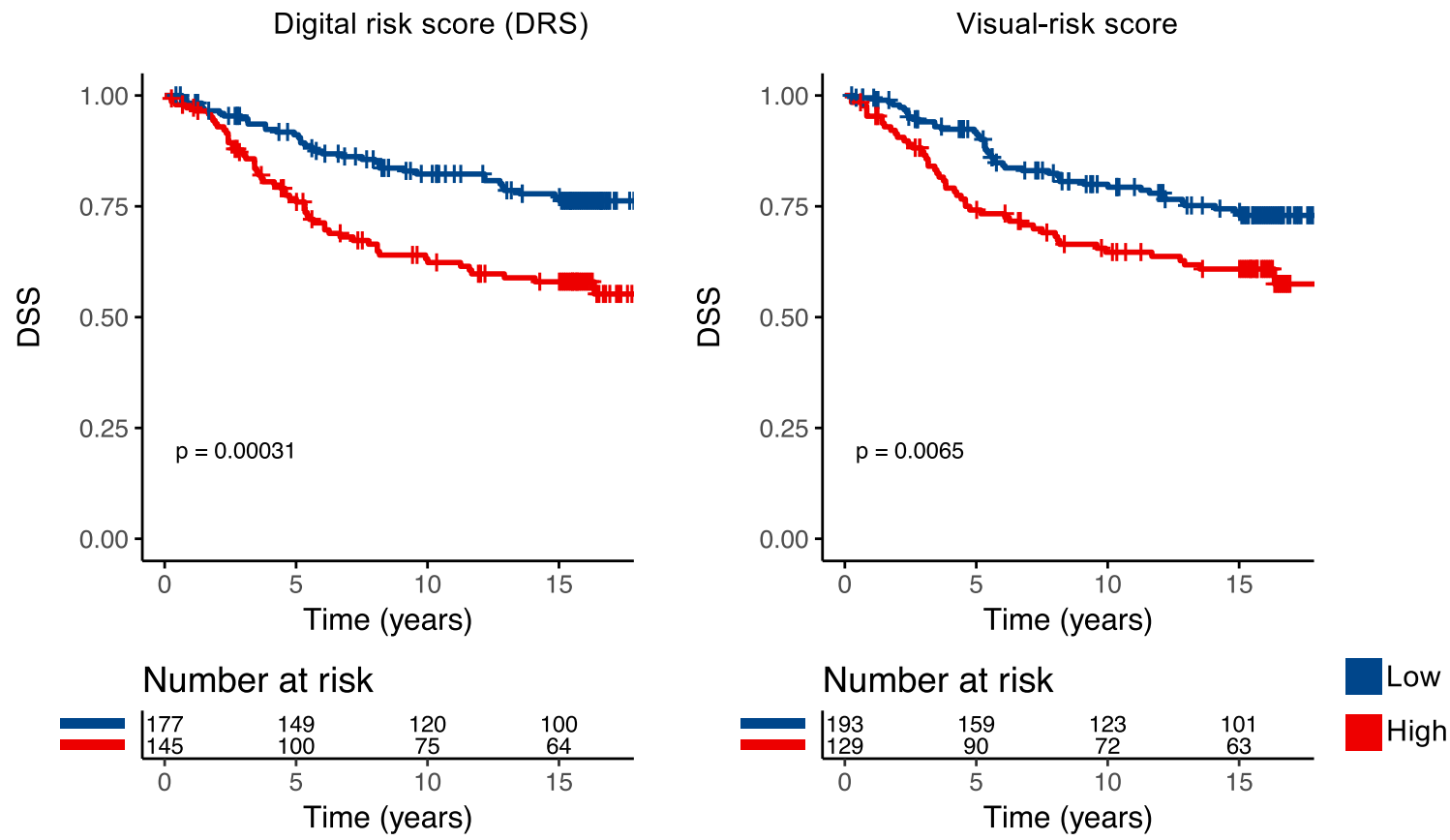

Fig. 2 Disease-specific survival (DSS) and overall survival (OS) according the classification into low and high digital risk score (DRS) groups

\section{Outcome classification and visual risk score}

Out of the 431 test TMA spot images, 109 were classified by at least one pathologist as not evaluable due to insufficient amount of cancer tissue or partial spot detachment for reliable risk assessment and were therefore left out from the analyses.

In the remaining subset of 322 spot images, $60 \%$ $(N=193)$ of the patients were assigned to the low-risk and $40 \%(N=129)$ to the high-risk group according the majority vote visual risk score, as compared to $55 \%(N=177)$ low risk and $45 \%(N=145)$ high risk according to the DRS groups. Percent agreement between the pathologists' individual scorings was $32 \%$. There was a significant agreement between pathologist 1 and $3\left(\kappa_{(1,3)}=0.27 ; p<0.001\right)$, but not between pathologist 1 and $2\left(\kappa_{(1,2)}=0.005 ; p=0.931\right)$ or pathologist 2 and $3\left(\kappa_{(2,3)}=-0.028 ; p=0.598\right)$ in assigning the patients into low- and high-risk groups.

In a univariate analysis, the digital risk score was found to be a significant predictor of disease-specific survival with a HR $=2.10$ (95\% CI 1.40-3.18, $p<0.001)$ and C-index of 0.60 (95\% CI 0.55-0.65). Similarly, the visual risk score was found to be a significant predictor of survival with a $\mathrm{HR}=1.74(95 \%$ CI $1.16-2.61, p=0.006)$ and C-index of 0.58 (95\% CI 0.53-0.63) (Supplementary Fig. 2). Interestingly, a Chi-square test of these univariate survival models indicated that the models were significantly different $(p<0.001)$. When the visual risk score and the DRS group were both included as covariables in a multivariate survival analysis, both turned out to be independent predictors $(\mathrm{HR}=2.05, p<0.001$ for the DRS and $\mathrm{HR}=1.68$, $p=0.012$ for the visual risk score). C-index of the combined survival model was 0.64 (95\% CI 0.58-0.69). An analysis of the association between cancer morphological features and the digital risk score showed that the DRS was significantly correlated with nuclear pleomorphism and tissue tubule formation, whereas the visual risk score was significantly associated also with cancer mitotic count, presence of necrosis and the number of TILs (Supplementary Table 1).

\section{Discussion}

We found that by utilising machine learning algorithms it is possible to extract information relevant for breast cancer patient outcomes from tumour tissue images stained for the basic morphology only. Importantly, the results show that prognostic discrimination is achievable without guidance or the use of prior knowledge of breast cancer biology or pathology in the training of the algorithm. Instead of directing the focus towards cancer cellular features (e.g. number of mitotic cells, immune cells, pleomorphic cells) or tissue entities (e.g. duct formation, presence of tumour necrosis), we guided the supervision simply with the patient survival outcome data.

Computerised methods for analysing breast tissue images for patient prognostication have been studied earlier. Extracting more than 6000 predefined image features from two 


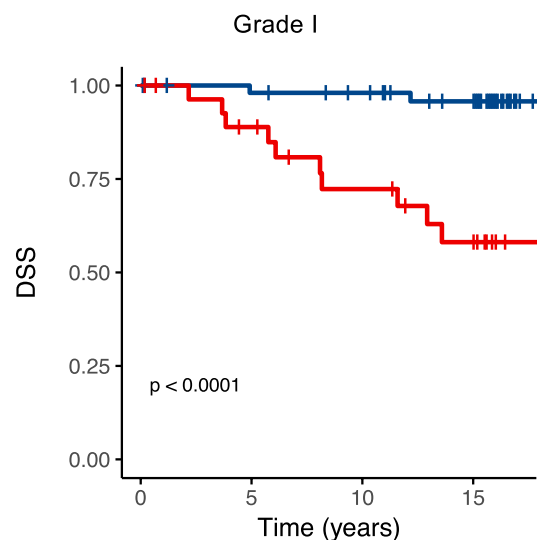

\section{Number at risk}

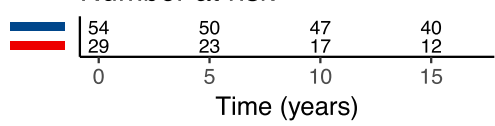

ER-

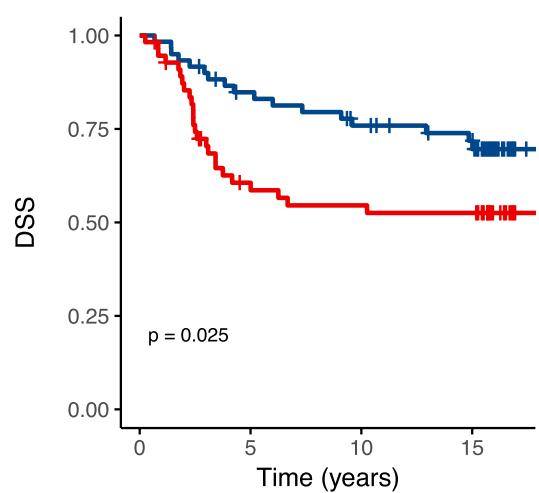

\section{Number at risk}

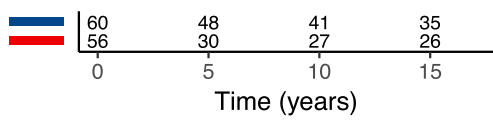

ER+

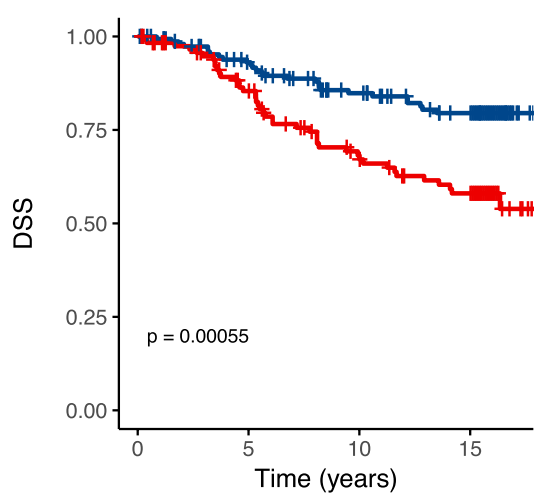

\section{Number at risk}

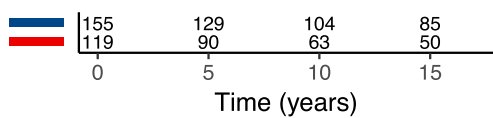

Grade II

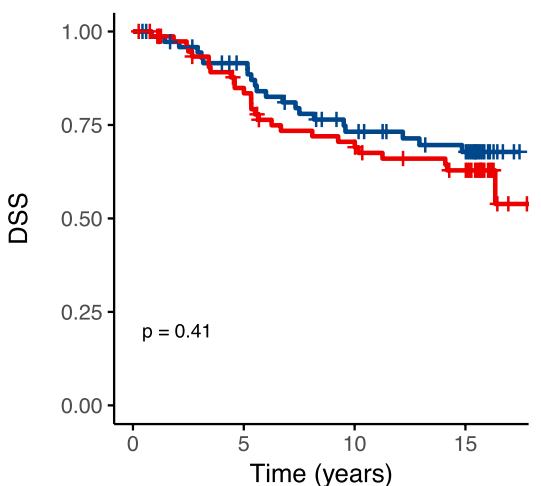

Number at risk

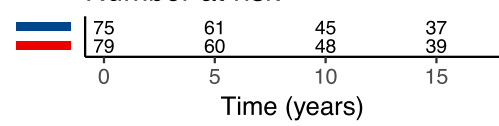

PR-

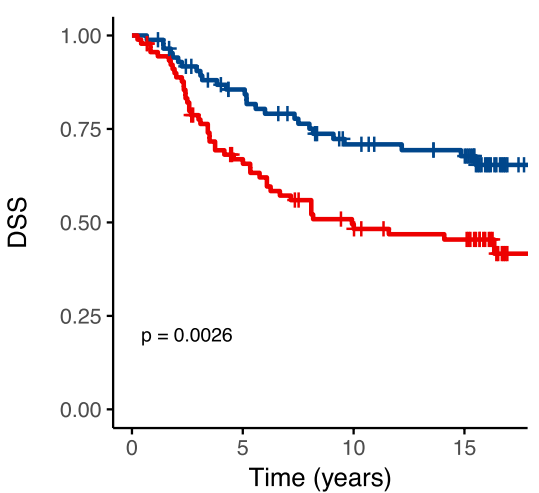

Number at risk

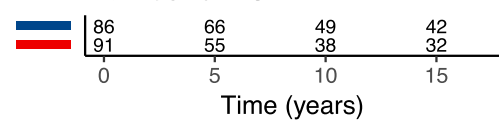

$\mathrm{PR}+$

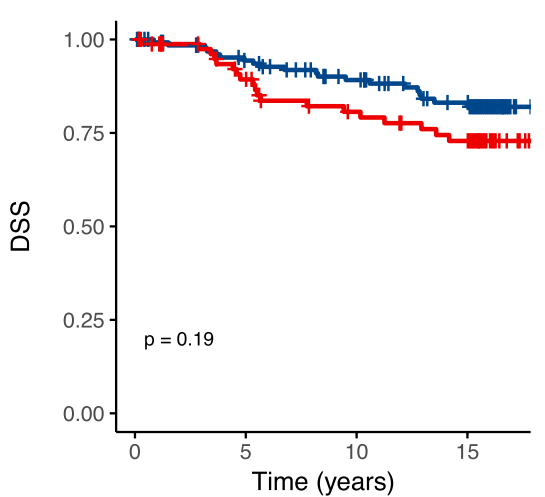

Number at risk

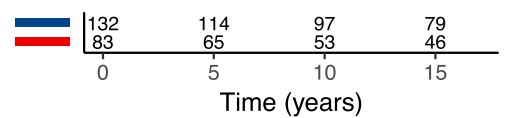

Grade III

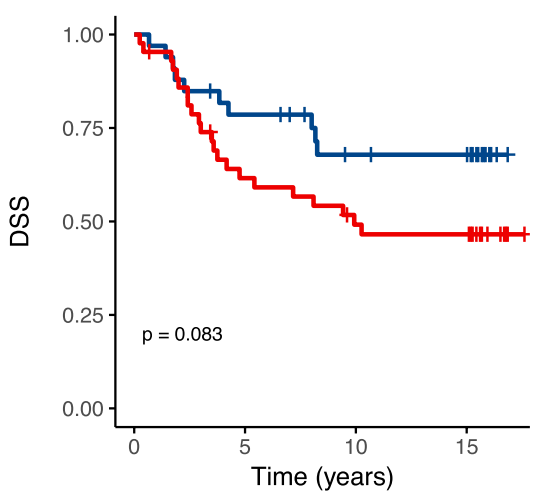

Number at risk

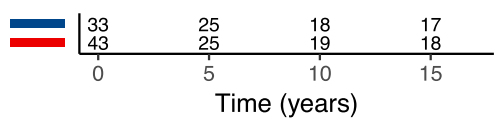

HER2-

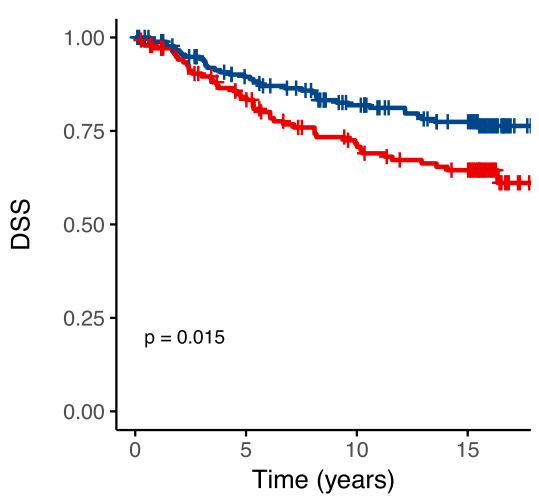

Number at risk

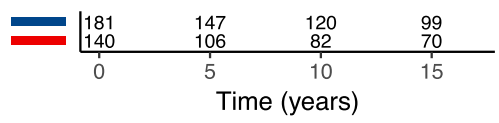

HER2+

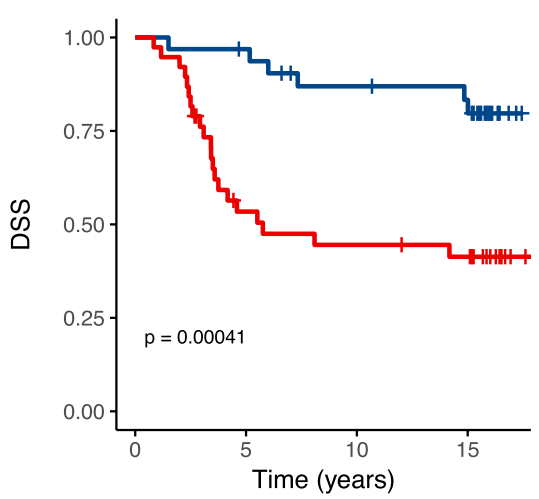

Number at risk

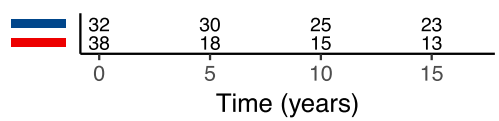

Digital risk score (DRS): $\quad$ Low High

Fig. 3 Disease-specific survival (DSS) according the classification into low and high digital risk score (DRS) groups 


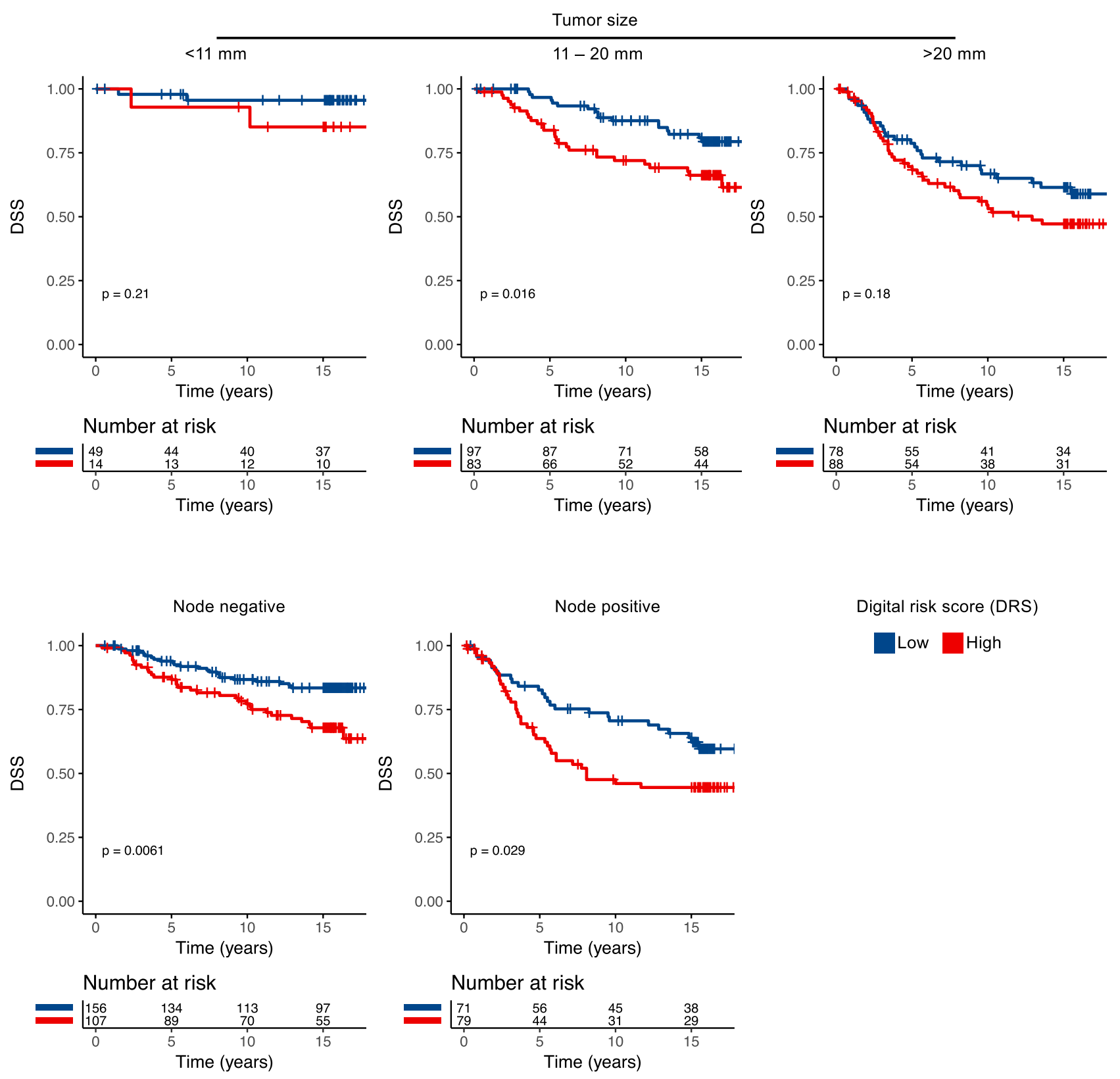

Fig. 4 Disease-specific survival (DSS) according the classification into low and high digital risk group (DRS) groups in patients with different tumour size and nodal status

cohorts ( $N=248$ and $N=328$ ), authors in [35] showed that it is feasible to learn an outcome predictor for overall survival ( $\mathrm{HR}=1.78, p=0.017)$ using small tumour regions. Using a subset of the whole slides from [36], an earlier study [37] proposed a joint analysis of image features and gene expression signatures for prognostic biomarker discovery. The authors used a training set of 131 patients and validated the biomarkers with H\&E-stained tumour samples from 65 breast cancer patients. The strongest predictive image feature the authors identified reached a HR $=1.7(p=0.002)$ in prediction of relapse-free survival. Moreover, a previous work
[38] identified morphological features in a data set of 230 breast cancer patients that were independent and prognostic for 8-year disease-free survival. Our study extends this body of work by demonstrating that it is possible to learn a prognostic signal from a patient cohort without domain knowledge. Our analysis was blinded from the fundamental concepts such as cells and nuclei, different tissue compartments and histological grade that were incorporated in the previous studies. Nevertheless, we were able to train an independent risk predictor based on the training cohort, using the raw image data and follow-up information only. Furthermore, we 
Table 2 Cox uni- and multivariate survival analysis

\begin{tabular}{|c|c|c|c|c|c|c|}
\hline \multirow[t]{2}{*}{ Variables } & \multicolumn{3}{|c|}{ Univariate analysis } & \multicolumn{3}{|c|}{ Multivariate analysis } \\
\hline & HR & CI $95 \%$ & $p$ value & $\mathrm{HR}$ & CI $95 \%$ & $p$ Value \\
\hline \multicolumn{7}{|l|}{ DRS } \\
\hline Low & Ref. & & & Ref. & & \\
\hline High & 2.10 & $(1.33-3.32)$ & 0.001 & 2.04 & $(1.20-3.44)$ & 0.007 \\
\hline \multicolumn{7}{|c|}{$\begin{array}{l}\text { Number of positive } \\
\text { lymph nodes }\end{array}$} \\
\hline 0 & Ref. & & & Ref. & & \\
\hline $1-3$ & 1.53 & $(0.89-2.63)$ & 0.123 & 2.12 & $(0.83-1.47)$ & 0.116 \\
\hline $4-9$ & 2.93 & $(1.61-5.33)$ & $<0.001$ & 2.15 & $(0.75-6.19)$ & 0.154 \\
\hline$>10$ & 7.43 & $(2.90-19.02)$ & $<0.001$ & 4.75 & $(1.17-19.30)$ & 0.029 \\
\hline \multicolumn{7}{|l|}{ Tumour size } \\
\hline Per mm & 1.04 & $(1.03-1.06)$ & $<0.001$ & 1.04 & $(1.02-1.06)$ & $<0.001$ \\
\hline \multicolumn{7}{|l|}{ Histological grade } \\
\hline I & Ref. & & & Ref. & & \\
\hline II or III & 3.14 & $(1.61-6.09)$ & $<0.001$ & 1.57 & $(0.76-3.20)$ & 0.220 \\
\hline \multicolumn{7}{|l|}{ Histological type } \\
\hline Ductal & Ref & & & Ref. & & \\
\hline Lobular/special & 0.73 & $(0.40-1.33)$ & 0.306 & 0.90 & $(0.41-1.95)$ & 0.782 \\
\hline \multicolumn{7}{|l|}{ Age } \\
\hline$\leq 39$ & Ref. & & & Ref. & & \\
\hline $40-49$ & 0.78 & $(0.33-1.88)$ & 0.585 & 0.43 & $(0.17-1.12)$ & 0.084 \\
\hline $50-59$ & 0.69 & $(0.28-1.70)$ & 0.425 & 0.48 & $(0.19-1.28)$ & 0.144 \\
\hline $60-69$ & 1.00 & $(0.42-2.36)$ & 0.996 & 0.62 & $(0.25-1.57)$ & 0.319 \\
\hline$\geq 70$ & 1.58 & $(0.66-3.79)$ & 0.306 & 1.35 & $(0.49-3.72)$ & 0.564 \\
\hline \multicolumn{7}{|l|}{ ER } \\
\hline Negative & Ref. & & & & & \\
\hline Positive & 0.69 & $(0.44-1.09)$ & 0.15 & & & \\
\hline \multicolumn{7}{|l|}{ PR } \\
\hline Negative & Ref. & & & Ref. & & \\
\hline Positive & 0.34 & $(0.21-0.55)$ & $<0.001$ & 0.42 & $(0.25-0.71)$ & 0.001 \\
\hline \multicolumn{7}{|l|}{ HER2 } \\
\hline Negative & Ref. & & & & & \\
\hline Positive & 1.51 & $(0.90-2.53)$ & 0.119 & 1.07 & $(0.57-1.98)$ & 0.831 \\
\hline \multicolumn{7}{|c|}{ Systematic therapy } \\
\hline Not given & Ref. & & & Ref. & & \\
\hline Given & 1.90 & $(1.21-2.98)$ & 0.005 & 1.07 & $(0.22-1.47)$ & 0.245 \\
\hline \multicolumn{7}{|l|}{ Local therapy } \\
\hline Not given & Ref. & & & Ref & & \\
\hline Given & 1.23 & $(0.75-2.03)$ & 0.404 & 1.22 & $(0.61-2.46)$ & 0.571 \\
\hline
\end{tabular}

Histological grade and type were assessed from whole tumour sections, while ER, PR and HER2 were assessed from TMAs. In order to meet the Cox proportionality assumption, ER was left out from the multivariate analysis and grade II and III were combined

$E R$ estrogen receptor status, $P R$ progesterone receptor status, HER2 human epidermal growth factor receptor 2 gene amplification

$p$-values $<0.05$ are shown in bold

used a large multicentre cohort with a median follow-up time of over 15 years and analysed the associations of the outcome predictor with the commonly used clinicopathological variables. Outside breast cancer, direct outcome prediction using tissue morphology has been successfully applied in colorectal cancer [39] and glioma [40].

Moreover, we compared the DRS group with the visual risk score, which combined three pathologists' risk assessments according to a majority vote rule. Even though 
pathologists do not perform such a direct risk assessment as part of breast cancer diagnostics, we wanted to evaluate the prognostic potential of morphological features detected by pathologists in a small tumour tissue area (a TMA core) and compare this with the corresponding digital risk score. The analysis indicated that the visual risk score was a significant predictor of outcome, but that the digital risk score yielded a slightly stronger discrimination than the visual risk score (C-index 0.60 vs. 0.58 ). As expected, the visual risk score correlated with known tissue entities (mitoses, pleomorphism, tubules, necrosis and TILs). Interestingly, the DRS group associated only with pleomorphism and tubules, indicating that the machine learning algorithm partly has learned known prognostic entities, but partly has extracted features that are not fully explained by known factors. This was supported by multivariate survival analysis with DRS and visual risk score, which showed increased discrimination (C-index 0.64), and revealed that the risk scores are independent prognostic factors.

One of the main reasons behind the success of deep learning and CNNs has been improved availability of large data sets [41, 42]. The best-performing CNNs for object detection and classification are trained with millions of images [43-45]. Contrary to classification based on handcrafted image descriptors and shallow learners, $\mathrm{CNN}$ inherently learns hierarchical image features from data, and larger data set usually leads into more powerful models. This ability to learn features directly from the data makes CNNs perform well and why they are easy to use. However, when only limited number of data points is available, direct end-to-end training of a CNN might not lead into any added benefit over handcrafted features and a shallow classifier.

Our goal in the design of the computational pipeline for patient outcome classification was to combine the best from the both worlds; the descriptive power of CNNs with the capability of shallow learners to generate robust models from more limited data set. Generally, this approach is known as transfer learning, which is a popular strategy to achieve a strong performance even with smaller data sets $[46,47]$. We took advantage of a CNN trained on the ImageNet [48], a large database for object recognition, and used it for extracting local image descriptors. An important benefit of this approach is less computational requirements, since training of the $\mathrm{CNN}$ is not needed. Furthermore, the approach is agnostic with regard to the $\mathrm{CNN}$ used and is easily amendable and compatible with novel model architectures frequently discovered and shared online for the research community. The ImageNet consists of photographs representing natural objects from bicycles to goldfish. ${ }^{2} \mathrm{His}-$ tological images are fundamentally different from everyday

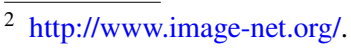

photos and it is reasonable to assume that the descriptors learned in natural images are not optimally suited for analysis of tumour tissue images. IFV is an orderless descriptor aggregation method, capturing the first- and second-order statistics of the GMM modes. The GMM modes were learned in the training set of tumour tissue images, and therefore this intermediate unsupervised learning phase further fine-tunes the features more suitable to the domain of histological images.

Our study has some important limitations. The cohort used in this study was centrally scanned using the same slide scanner and therefore the generalisation of the outcome prediction to tissue images from other slide scanners was not taken into consideration. Moreover, our study considered only small tumour area in the form of a TMA spot image.

Although our analysis indicated correlation with the computerised prediction and pleomorphism and tubules, a major limitation of the current work is the difficulty to explain the exact source and location of the predictive signal, i.e. which tissue regions gave rise to the result obtained. Deep learning models are considered as "black boxes", which work well, but whose function, or reasoning, is difficult to reveal [49]. Some approaches to answer this shortcoming have been presented [50], but this is an active research question in field of machine learning and no direct solution for this exists at present. We intend to address this in the future studies.

Our findings indicate that computerised methods offer an innovative approach for analysing histological samples. Nevertheless, future studies are required to validate our findings, test similar algorithms on larger data sets representing different malignancies.

\section{Conclusions}

We have demonstrated how machine learning analysis of tumour tissue images can be utilised for breast cancer patient prognostication. Our results show that it is possible to learn a risk grouping, providing independent prognostic value complementing the conventional clinicopathological variables, using only digitised tumour tissue images and patient outcome as the endpoint. These findings suggest that machine learning algorithms together with large-scale tumour tissue image series may help approximate the full prognostic potential of tumour morphology.

Acknowledgements Open access funding provided by University of Helsinki including Helsinki University Central Hospital. We thank the Digital Microscopy and Molecular Pathology unit at FIMM, supported by the Helsinki Institute of Life Science and Biocenter Finland for providing slide scanning services.

Author contributions Conception and design: RT, NL, JL. Development of the methodology: RT. Acquisition of data: ML, PEK, SN, CV. 
Analysis and interpretation of data: RT, NL, JL. Writing, review and/ or revision of the manuscript: RT, SN, HJ, NL, JL. Administrative, technical or material support: DB, ML, JI, KvS, HJ. Study supervision: NL, JL.

Funding Biomedicum Helsinki Foundation, Orion-Pharmos Research Foundation, Cancer Society of Finland, Emil Aaltonen Foundation, Ida Montin Foundation, Doctoral Program in Biomedicine, Sigrid Juselius Foundation, Helsinki Institute of Life Science Fellowship Program, Biocenter Finland and the National Institute for Health Research (NIHR) Oxford Biomedical Research Centre (BRC) (Molecular Diagnostics Theme/Multimodal Pathology Subtheme).

Data availability The data that support the findings of this study are available from the University of Helsinki but restrictions apply to the availability of these data, which were used under license for the current study, and so are not publicly available. Data are however available from the authors upon reasonable request and with permission of University of Helsinki.

\section{Compliance with ethical standards}

Conflict of interest Johan Lundin and Mikael Lundin are founders and co-owners of Aiforia Technologies Oy, Helsinki, Finland. Other authors have no conflict of interest.

Ethics approval Project-specific ethical approval for the use of clinical samples and retrieving clinical data was approved by the local operative ethics committee of the Hospital District of Helsinki and Uusimaa (DNo 94/13/03/02/2012). Also, clearance from the National Supervisory Authority for Welfare and Health, Valvira, for using human tissues for research, has been approved (DNo 7717/06.01.03.01/2015).

Open Access This article is distributed under the terms of the Creative Commons Attribution 4.0 International License (http://creativeco mmons.org/licenses/by/4.0/), which permits unrestricted use, distribution, and reproduction in any medium, provided you give appropriate credit to the original author(s) and the source, provide a link to the Creative Commons license, and indicate if changes were made.

\section{References}

1. Obermeyer Z, Emanuel EJ (2016) Predicting the future-big data, machine learning, and clinical medicine. N Engl J Med 375:12161219. https://doi.org/10.1056/NEJMp1606181

2. Schmidhuber J (2015) Deep learning in neural networks: an overview. Neural Netw 61:85-117. https://doi.org/10.1016/j.neune t.2014.09.003

3. LeCun Y, Bengio Y, Hinton G (2015) Deep learning. Nature 521:436-444. https://doi.org/10.1038/nature14539

4. Shen D, Wu G, Suk H-I (2017) Deep learning in medical image analysis. Annu Rev Biomed Eng 19:221-248. https://doi. org/10.1146/annurev-bioeng-071516-044442

5. Litjens G, Kooi T, Bejnordi BE et al (2017) A survey on deep learning in medical image analysis. Med Image Anal 42:60-88. https://doi.org/10.1016/j.media.2017.07.005

6. Gulshan V, Peng L, Coram M et al (2016) Development and validation of a deep learning algorithm for detection of diabetic retinopathy in retinal fundus photographs. JAMA 316:2402. https ://doi.org/10.1001/jama.2016.17216
7. Esteva A, Kuprel B, Novoa RA et al (2017) Dermatologist-level classification of skin cancer with deep neural networks. Nature 542:115-118. https://doi.org/10.1038/nature21056

8. Madabhushi A, Lee G (2016) Image analysis and machine learning in digital pathology: challenges and opportunities. Med Image Anal 33:170-175. https://doi.org/10.1016/j.media.2016.06.037

9. Cireşan DC, Giusti A, Gambardella LM, Schmidhuber J (2013) Mitosis detection in breast cancer histology images with deep neural networks. Med Image Comput Comput Assist Interv 16:411-418

10. Paeng K, Hwang S, Park S, Kim M (2017) A unified framework for tumor proliferation score prediction in breast histopathology. Springer, Cham, pp 231-239

11. Veta M, van Diest PJ, Willems SM et al (2015) Assessment of algorithms for mitosis detection in breast cancer histopathology images. Med Image Anal 20:237-248. https://doi.org/10.1016/j. media.2014.11.010

12. Turkki R, Linder N, Kovanen PE et al (2016) Antibody-supervised deep learning for quantification of tumor-infiltrating immune cells in hematoxylin and eosin stained breast cancer samples. J Pathol Inform. https://doi.org/10.4103/21533539.189703

13. Basavanhally AN, Ganesan S, Agner S et al (2010) Computerized image-based detection and grading of lymphocytic infiltration in HER2 + breast cancer histopathology. IEEE Trans Biomed Eng 57:642-653. https://doi.org/10.1109/TBME.2009.2035305

14. Litjens G, Sánchez CI, Timofeeva N et al (2016) Deep learning as a tool for increased accuracy and efficiency of histopathological diagnosis. Sci Rep 6:26286. https://doi.org/10.1038/srep26286

15. Xu J, Luo X, Wang G et al (2016) A deep convolutional neural network for segmenting and classifying epithelial and stromal regions in histopathological images. Neurocomputing 191:214223. https://doi.org/10.1016/j.neucom.2016.01.034

16. Chen H, Qi X, Yu L et al (2017) DCAN: deep contour-aware networks for object instance segmentation from histology images. Med Image Anal 36:135-146. https://doi.org/10.1016/j.media .2016.11.004

17. Turkki R, Linder N, Holopainen T et al (2015) Assessment of tumour viability in human lung cancer xenografts with texturebased image analysis. J Clin Pathol 68:jclinpath-2015. https://doi. org/10.1136/jclinpath-2015-202888

18. Roxanis I, Colling R, Kartsonaki C et al (2018) The significance of tumour microarchitectural features in breast cancer prognosis: a digital image analysis. Breast Cancer Res 20:11. https://doi. org/10.1186/s13058-018-0934-x

19. Robertson S, Azizpour H, Smith K, Hartman J (2017) Digital image analysis in breast pathology-from image processing techniques to artificial intelligence. Transl Res 45:78. https://doi. org/10.1016/j.trsl.2017.10.010

20. Fukushima K (1980) Neocognitron: a self-organizing neural network model for a mechanism of pattern recognition unaffected by shift in position. Biol Cybern 36:193-202. https://doi. org/10.1007/BF00344251

21. Bejnordi BE, Veta M, Johannes van Diest P et al (2017) Diagnostic assessment of deep learning algorithms for detection of lymph node metastases in women with breast cancer. JAMA 318:2199. https://doi.org/10.1001/jama.2017.14585

22. Joensuu $\mathrm{H}$, Isola $J$, Lundin $M$ et al (2003) Amplification of erbB2 and erbB2 expression are superior to estrogen receptor status as risk factors for distant recurrence in pT1N0M0 breast cancer: a nationwide population-based study. Clin Cancer Res 9:923-930

23. Kononen J, Bubendorf L, Kallioniemi A et al (1998) Tissue microarrays for high-throughput molecular profiling of tumor specimens. Nat Med 4:844-847

24. Tavassoéli F, Devilee P (eds) (2003) Pathology and genetics of tumours of the breast and female genital organs. WHO, Geneva 
25. Simonyan K, Zisserman A (2015) Very deep convolutional networks for large-scale image recognition. In: International conference on learning representations

26. Perronnin F, Sánchez J, Mensink T (2010) Improving the Fisher Kernel for large-scale image classification. Springer, Berlin, pp $143-156$

27. Cimpoi M, Maji S, Kokkinos I, Vedaldi A (2016) Deep filter banks for texture recognition, description, and segmentation. Int J Comput Vis. https://doi.org/10.1007/s11263-015-0872-3

28. Chen Yu, Chen Dian-ren, Li Yang, Chen Lei (2010) Otsu's thresholding method based on gray level-gradient two-dimensional histogram. In: Proceedings of the IEEE 2010 2nd international asia conference on informatics in control, automation and robotics (CAR 2010). pp 282-285

29. Fan R-E, Chang K-W, Hsieh C-J et al (2008) LIBLINEAR: a library for large linear classification. J Mach Learn Res 9:1871-1874

30. Vedaldi A, Fulkerson B (2010) VLFeat: an open and portable library of computer vision algorithms. In: Proceedings of the international conference on multimedia. ACM, pp 1469-1472

31. Vedaldi A, Lenc K (2015) MatConvNet-convolutional neural networks for MATLAB. In: Proceedings of the 23rd ACM international conference on multimedia, Brisbane, Australia, October 26-30, 2015

32. Kaplan EL, Meier P (1958) Nonparametric estimation from incomplete observations. J Am Stat Assoc 53:457. https://doi. org/10.2307/2281868

33. Cox DR (1972) Regression models and life-tables. J R Stat Soc Ser B 34:187-220. https://doi.org/10.2307/2985181

34. Gönen M, Heller G (2005) Concordance probability and discriminatory power in proportional hazards regression. Biometrika 92:965-970. https://doi.org/10.1093/biomet/92.4.965

35. Beck AH, Sangoi AR, Leung S et al (2011) Systematic analysis of breast cancer morphology uncovers stromal features associated with survival. Sci Transl Med 3:108ra113. https://doi.org/10.1126/ scitranslmed.3002564

36. Moor AE, Guevara C, Altermatt HJ et al (2011) PRO_10-a new tissue-based prognostic multigene marker in patients with early estrogen receptor-positive breast cancer. Pathobiology 78:140148. https://doi.org/10.1159/000323809

37. Popovici V, Budinská E, Čápková L et al (2016) Joint analysis of histopathology image features and gene expression in breast cancer. BMC Bioinf 17:209. https://doi.org/10.1186/s1285 9-016-1072-Z

38. Chen J-M, Qu A-P, Wang L-W et al (2015) New breast cancer prognostic factors identified by computer-aided image analysis of HE stained histopathology images. Sci Rep 5:10690. https://doi. org/10.1038/srep10690
39. Bychkov D, Linder N, Turkki R et al (2018) Deep learning based tissue analysis predicts outcome in colorectal cancer. Sci Rep 8:3395. https://doi.org/10.1038/s41598-018-21758-3

40. Mobadersany P, Yousefi S, Amgad M et al (2018) Predicting cancer outcomes from histology and genomics using convolutional networks. Proc Natl Acad Sci USA 115:E2970-E2979. https://doi. org/10.1073/pnas.1717139115

41. Sun C, Shrivastava A, Singh S, Gupta A (2017) Revisiting unreasonable effectiveness of data in deep learning era. In: 2017 IEEE International conference on computer vision (ICCV), Venice, Italy, 22-29 October 2017, pp 843-852

42. Joulin A, van der Maaten L, Jabri A, Vasilache N (2016) Learning visual features from large weakly supervised data. In: Leibe B, Matas J, Sebe N, Welling M (eds) Computer vision - ECCV 2016. ECCV 2016. Lecture notes in computer Science, vol 9911. Springer, Cham

43. Szegedy C, Wei Liu, Yangqing Jia, et al (2015) Going deeper with convolutions. In: Proceedings of the 2015 IEEE conference on computer vision and pattern recognition (CVPR). pp 1-9

44. Krizhevsky A, Sutskever I, Hinton GE (2012) Imagenet classification with deep convolutional neural networks. Commun ACM 60:84-90

45. He K, Zhang X, Ren S, Sun J (2015) Deep residual learning for image recognition. In: 2016 IEEE conference on computer vision and pattern recognition. pp 770-778

46. Razavian AS, Azizpour H, Sullivan J, Carlsson S (2014) CNN features off-the-shelf: an astounding baseline for recognition. In: Proceedings 2014 IEEE conference on computer vision and pattern recognition workshops. pp 512-519

47. Yosinski J, Clune J, Bengio Y, Lipson H (2014) How transferable are features in deep neural networks? pp 3320-3328

48. Socher R (2009) ImageNet: a large-scale hierarchical image database. In: Proceedings 2009 IEEE conference on computer vision and pattern recognition. pp 248-255

49. Voosen P (2017) The AI detectives. Science 357:22-27. https:// doi.org/10.1126/science.357.6346.22

50. Montavon G, Lapuschkin S, Binder A et al (2017) Explaining nonlinear classification decisions with deep Taylor decomposition. Pattern Recognit 65:211-222. https://doi.org/10.1016/j.patco g.2016.11.008

Publisher's Note Springer Nature remains neutral with regard to jurisdictional claims in published maps and institutional affiliations. 